\title{
Simulation of Buckling and Dead Lock States of Drill Strings in Curvilinear Bore-Holes
}

\author{
[ V.I. Gulyayev, S.N. Glazunov, O.M. Andrusenko, N.V. Shlun ]
}

\begin{abstract}
This paper deals with simulating the regimes of drilling deep curvilinear bore-holes with prescribed geometrical imperfections of their axial lines. The interpolation techniques (spline approximation) are used for the well shape representation. On the basis of the theory of curvilinear flexible elastic rods, the 3D "stiff-string" drag and torque model of the drill string bending is elaborated. With its application it is shown that the contact and friction forces generated by interaction of the drill string with the bore-hole surface can be calculated and regulate, after their analysis, the regimes of buckling and dead lock states of drill strings in the curvilinear cavities of the boreholes are simulated.
\end{abstract}

Keywords-bore-hole channel, elastic drill string, contact forces, resistance forces, critical buckling

\section{Introduction}

Mastering the technology of drilling deep oil and gas boreholes is one of the most important problems of modern mining industry. Leading position in this direction belongs to the rotor method of drilling. With its use the technology of industrial drilling the bore-holes $6 \mathrm{~km}$ in depth is worked out and the purpose of drilling $7 \mathrm{~km}$ bore-holes and deeper is posed [9]. The operating conditions of (dead lock states [1, 2, 4, 6, 7] and their driving can be accompanied by the phenomena of bifurcational buckling $[3,5,8]$ of the drill strings as well as self-excitation of vibrations of their bits. However up to now there are not reliable methods of physical and mathematical simulation of the pointed out effects.

Taking into consideration that mechanical occurrence attending these processes are very complicated and there is no producing experience of such wells drivage, it may be concluded that the problems of their theoretical simulation are topical.

V.I. Gulyayev, Dr. Sc., Professor, Head of Department of Mathematics National Transport University

Ukraine

S.N. Glazunov, Post-graduate student

O.M. Andrusenko, Ph. D., Corresponding author

N.V. Shlun, asistant

At the present time, the vertical, inclined and horizontal bore wells (Fig. 1) are drilled in accordance with requirements of economy, demands of oil-gas industry and its technological possibilities. Great attention is paid to the questions of drilling deep wells from ground surface and sea bottom. In the drilling technology the dominant role belongs to the rotor method based on the use of a drill string (DS) with a bit.

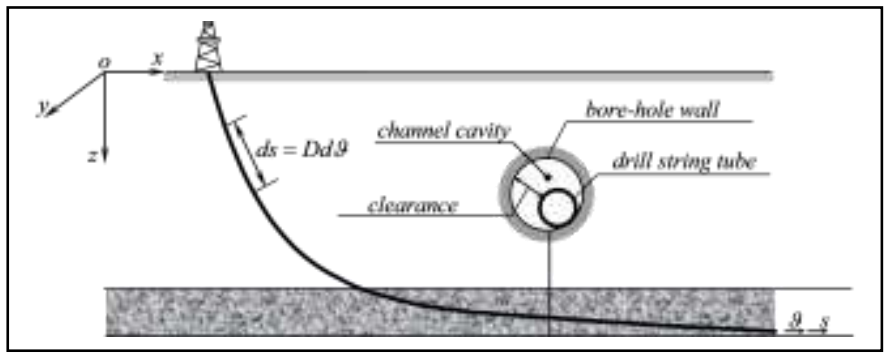

Figure 1. Curvilinear bore-hole scheme.

When the fuel extraction is realized from great depths, the drill efficiency is associated with the problems of revealing the emergency regimes of the DS functioning. The most drastic phenomena inducing the appearance of emergency situations during drilling are generation of intensive friction forces between the DS and bore-hole wall, loss of the DS mobility in the bore-hole channel and its critical buckling inside the channel cavity. Inasmuch as the drill string represents a very flexible rod due to its large length, it can transit from a stationary state of axial movement to the mode of locking at violation of the low friction conditions.

In a general case these phenomena constitute the motions of non-linear flexible rods which are performed under action of driving, gravity, contact, and friction forces. For their generating to happen, the non-linear contact and friction force interaction between separate parts of the system is of importance which regulates income and expenditure of the energy and by this means gives raise to emergency situation. In drill assemblies the reason of the buckling triggering is bifurcation disturbance of the balance between elastic forces in the DS, gravity forces and the forces of contact and friction interaction of the DS with the well wall. The major factor adding complexity to the problem of the phenomenon simulation is that the equation describing this process belongs to the singularly perturbed type and has the solutions in the shapes of internal and boundary wavelets.

By now even general characteristics of this phenomena are not known because the force and kinematic properties of the system depends on many parameters and it is difficult to trace their influences. So our interest is in establishment of overall regularities of the critical emergency onset and to find the factors exerting primary control over the process of the DS deforming. 


\section{Stiff-String Drag \& Torque Model for Curvilinear Channel}

Consider theoretic aspects of the problem of computer simulation of an elastic DS dragging inside a curvilinear borehole channel. Let the design bore-hole axis line be described by the equation

$$
\boldsymbol{\rho}=\boldsymbol{\rho}(s)
$$

in the Cartesian coordinate system Oxyz. Here $\boldsymbol{\rho}$ is the radius-vector $\boldsymbol{\rho}=x \mathbf{i}+y \mathbf{j}+z \mathbf{k} ; \mathbf{i}, \mathbf{j}, \mathbf{k}$ are the appropriate unit vectors; $s$ is the parameter determined by the length of the axial line from some initial point till the current one; $x(s)$, $y(s), z(s)$ are the differentiable functions.

The axial lines of the DS and bore-hole are considered to coincide.

To determine the bending stress-strain state of a DS, one must know the basics of geometric parameters of its axis like the curvature

$$
k_{R}=1 / R=\left|\boldsymbol{\rho}^{\prime \prime}\right|
$$

and torsion

$$
k_{T}=\boldsymbol{\rho}^{\prime}\left(\boldsymbol{\rho}^{\prime \prime} \times \boldsymbol{\rho}^{\prime \prime \prime}\right) /\left(\boldsymbol{\rho}^{\prime \prime} \boldsymbol{\rho}^{\prime \prime}\right)
$$

Here, the symbol prime designates the derivative with respect to $s, R$ is the curvature radius.

To deduce the constitutive equations of elastic deforming a curvilinear rod, a concomitant reference frame moving along its axial line with parameter $s$ change should be introduced. Study of these equations is most convenient with the use of the Frenet natural trihedron with unit vectors of the principal normal $\mathbf{n}$, binormal $\mathbf{b}$ and tangent $\boldsymbol{\tau}$.

At any cross-section of the DS, the principal vectors of internal forces $\mathbf{F}(s)$ and moments $\mathbf{M}(s)$ and the vectors of distributed external forces $\mathbf{f}(s)$ and moments $\mathbf{m}(s)$ satisfy the following equilibrium equations

$$
\frac{d \mathbf{F}}{d s}=-\mathbf{f}, \quad \frac{d \mathbf{M}}{d s}=-\boldsymbol{\tau} \times \mathbf{F}-\mathbf{m}
$$

Since the Frenet reference frame rotates with its moving along the DS axis, the total derivatives $d \mathbf{F} / d s, d \mathbf{M} / d s$ should be expressed in the following form

$$
\frac{d \mathbf{F}}{d s}=\frac{\tilde{d} \mathbf{F}}{d s}+\mathbf{\Omega} \times \mathbf{F}, \quad \frac{d \mathbf{M}}{d s}=\frac{\tilde{d} \mathbf{M}}{d s}+\mathbf{\Omega} \times \mathbf{M}
$$

Here, $\tilde{d} \ldots / d s$ is the local derivative; $\boldsymbol{\Omega}$ is the Darboux vector, determined through the expression

$$
\boldsymbol{\Omega}=k_{R} \mathbf{b}+k_{T} \boldsymbol{\tau}
$$

With allowance made for Eqs. (6), one can represent Eqs. (4) as follows

$$
\frac{\tilde{d} \mathbf{F}}{d s}=-\mathbf{\Omega} \times \mathbf{F}-\mathbf{f}, \quad \frac{\tilde{d} \mathbf{M}}{d s}=-\mathbf{\Omega} \times \mathbf{M}-\boldsymbol{\tau} \times \mathbf{F}-\mathbf{m}
$$

Consider that the process of the DS dragging inside the bore-hole channel is operated with constant axial velocity $a$ and rotation velocity $\omega$. Then, the external distributed forces $\mathbf{f}(s)$ and moments $\mathbf{m}(s)$ can be represented by the equalities

$$
\mathbf{f}=\mathbf{f}^{g r}+\mathbf{f}^{c}+\mathbf{f}^{f r}+\mathbf{f}^{i n}, \quad \mathbf{m}=\mathbf{m}^{f r}=m_{\tau}^{f r} \boldsymbol{\tau}
$$

Here, $\mathbf{f}^{g r}$ is gravity, $\mathbf{f}^{c}$ is the force of contact interaction between the surfaces of the DS and bore-hole, $\mathbf{f}^{f r}$ is the force of friction interaction between these surfaces, $\mathbf{f}^{\text {in }}$ is the inertia force, $\mathbf{m}^{f r}$ is the distributed moment of friction forces.

With the aid of correlations (2) - (7) one can transform Eqs. (8) to a scalar form and apply it to computer simulation of the DS dragging inside the curvilinear channel of a bore hole.

Represent Eqs. (8) in the scalar form [6]

$$
\begin{aligned}
& \frac{d F_{n}}{d s}=-k_{R} F_{\tau}+k_{T} F_{b}-f_{n}^{g r}-f_{n}^{c}, \\
& \frac{d F_{b}}{d s}=-k_{T} F_{n}-f_{b}^{g r}-f_{b}^{c}, \\
& \frac{d F_{\tau}}{d s}=k_{R} F_{n}-f_{\tau}^{g r}-f_{\tau}^{f r}, \\
& 0=-k_{R} M_{\tau}+A k_{R} k_{T}+F_{b}, \\
& \frac{d k_{R}}{d s}=-\frac{1}{A} F_{n}, \\
& \frac{d M_{\tau}}{d s}=-m_{\tau}^{f r} .
\end{aligned}
$$

In this system, four functions of internal force factors $F_{n}(s), F_{b}(s), F_{\tau}(s), M_{\tau}(s)$ are unknown. The external distributed forces $f_{n}^{c}(s), f_{b}^{c}(s), f_{\tau}^{f r}(s)$, and moment $m_{\tau}^{f r}(s)$ are also required, while the gravity forces $f_{n}^{g r}(s)$, $f_{b}^{g r}(s)$ are considered to be known. 


\section{Simulation of the Drill String Dragging with Rotation}

It is of interest to note that the possibility arises to regulate the resistance forces and moments, choosing different ratios $v$ between the velocities of axial $(\dot{w})$ and rotary $(\omega)$ motions of the DS. To illustrate this feature the inclined borehole $4000 \mathrm{~m}$ in depth and $8000 \mathrm{~m}$ in lateral deflection was considered for the values $v=100,10,5,1,0.1$, and 0 . In Figs. 2 and Fig. 3 the diagrams of axial forces $F_{w}(s)$ and torque $M_{w}(s)$ are shown.

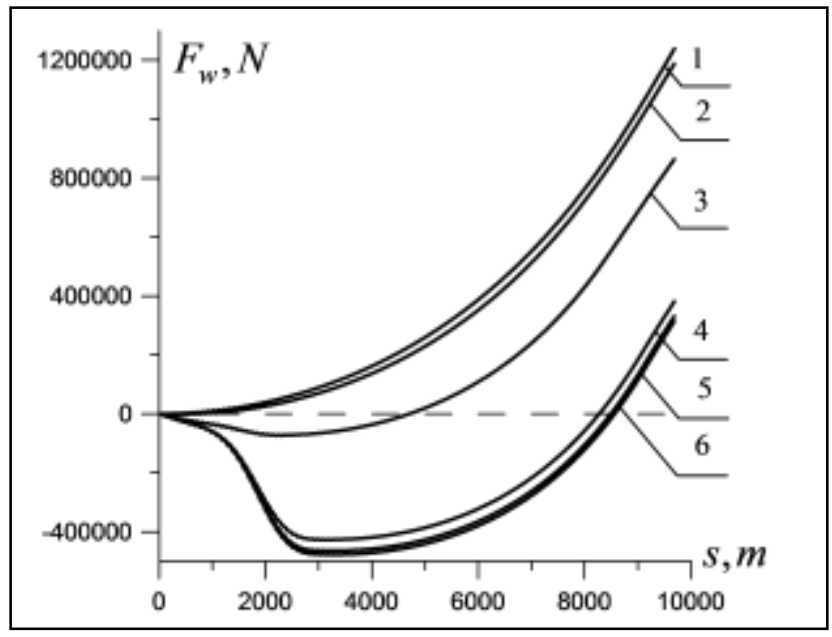

Figure 2. Function of axial force $F_{w}(s)$ in the drill string.

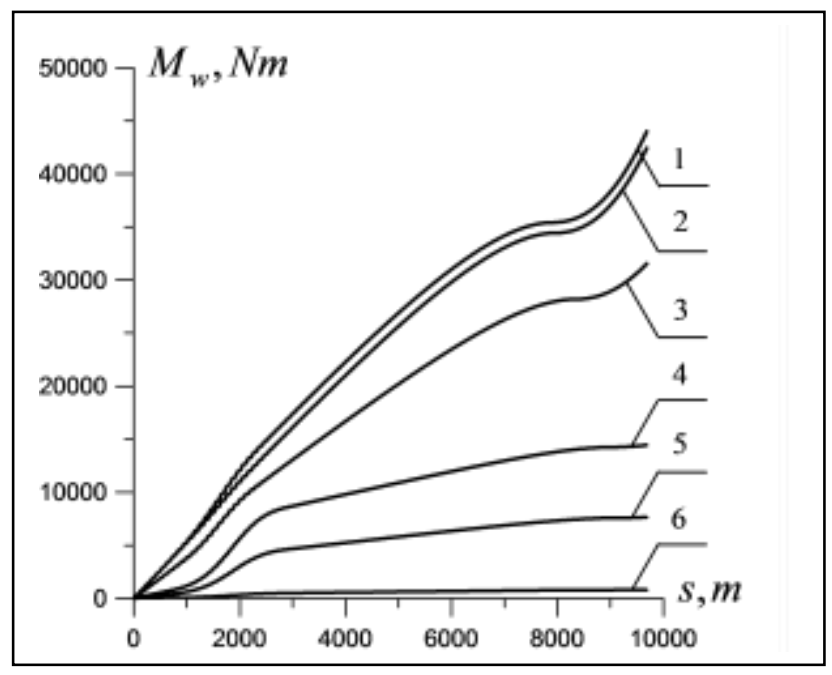

Figure 3. Function of tortque $M_{w}(s)$ in the drill string.

Positions 1 correspond to case of ideal geometry of the bore-hole and value $v=100$, positions $2-7$ are related to the bore-hole with geometric imperfection. The tripping in operations were analyzed in the bore. It can be seen that with the $v$ reduction the friction forces enlarge and axial compressing forces $F_{w}(s)$ appear in the drill string which can initiated its buckling.

Besides, the excessive compressing forces $F_{w}(s)$ may be responsible for appearance of the dead lock states.

\section{Bifurcational Correlations on the DS Buckling in a Curvilinear Bore-Hole}

To deduce constitutive equations of buckling the drill string in the channel of a curvilinear bore-hole, represent Eqs. (7) in moving reference frame oxyz with unit vectors $\mathbf{i}, \mathbf{j}, \mathbf{k}$ and linearize them in the vicinity of the considered state. Let the DS be lowering in a plane circular bore-hole. Then, it slides along the bore-hole bottom line and its axis is a circle of radius $\rho+a$, where $\rho$ is the radius of the bore-hole axis, "a" is the system clearance. Assume that the unknown variables got small increments marked by variation symbol $\delta$. Then, the non-linear equations (9) can be reduced to the homogeneous system

$$
\begin{aligned}
& \frac{d}{d s} \delta F_{y}=F_{z} \delta k_{x}-f^{g r} \sin \left(u_{0}+\frac{s}{\rho+a}\right) \delta v+ \\
& f^{g r} a \cos \left(u_{0}+\frac{s}{\rho+a}\right) \delta v^{\prime}, \\
& \frac{d}{d s} \delta v=\delta v^{\prime}, \\
& \frac{d}{d s} \delta v^{\prime}=-\frac{1}{a(\rho+a)} \delta v+\frac{1}{a} \delta k_{x}, \\
& \frac{d}{d s} \delta k_{x}=\frac{1}{E I} \delta F_{y} .
\end{aligned}
$$

where $u$ and $v$ are the coordinate variables on the channel surface $\Sigma$. Coefficients of Eqs. (10) are determined by the function of axial force $F_{z}(s)$ which can be constructed with the use of the techniques outlined in Parts II and III. Through variation of this function, it is possible to select its outlines, transforming Eqs. (10) to a degenerated form. These outlines of the $F_{z}(s)$ function are assumed to be critical and bifurcational, corresponding to the DS buckling.

In doing so, the non-trivial solution of Eqs. (10) represents the mode of stability loss of the DS.

\section{v. Results of the DS Buckling Analysis}

With the help of the elaborated approach, the effects of the DS buckling, occurring in rectilinear, circular, and curvilinear channels, were simulated. It was demonstrated that 
that the constructed constitutive equations, describing the buckling process, belong to the singularly perturbed class, therefore their solutions (and the buckling models) represent harmonic wavelets located) inside the DS length or in its boundary vicinity.

The modes of the DS buckling in circular bore-holes with asymmetric and symmetric outlines are shown in Figs. 4 and 5 , appropriately.

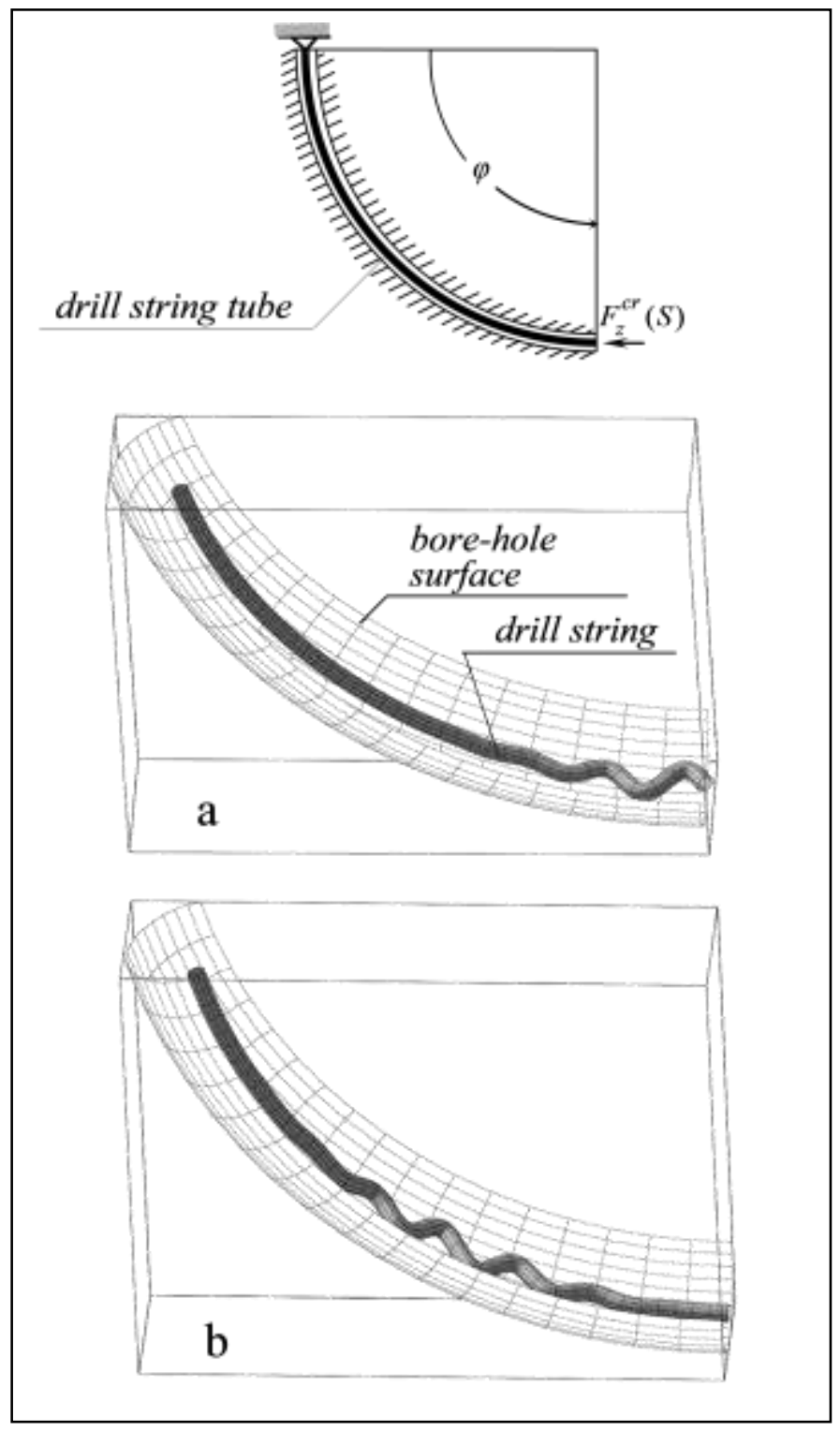

Figure 4. Drill string buckling in the asymmetric circular channel

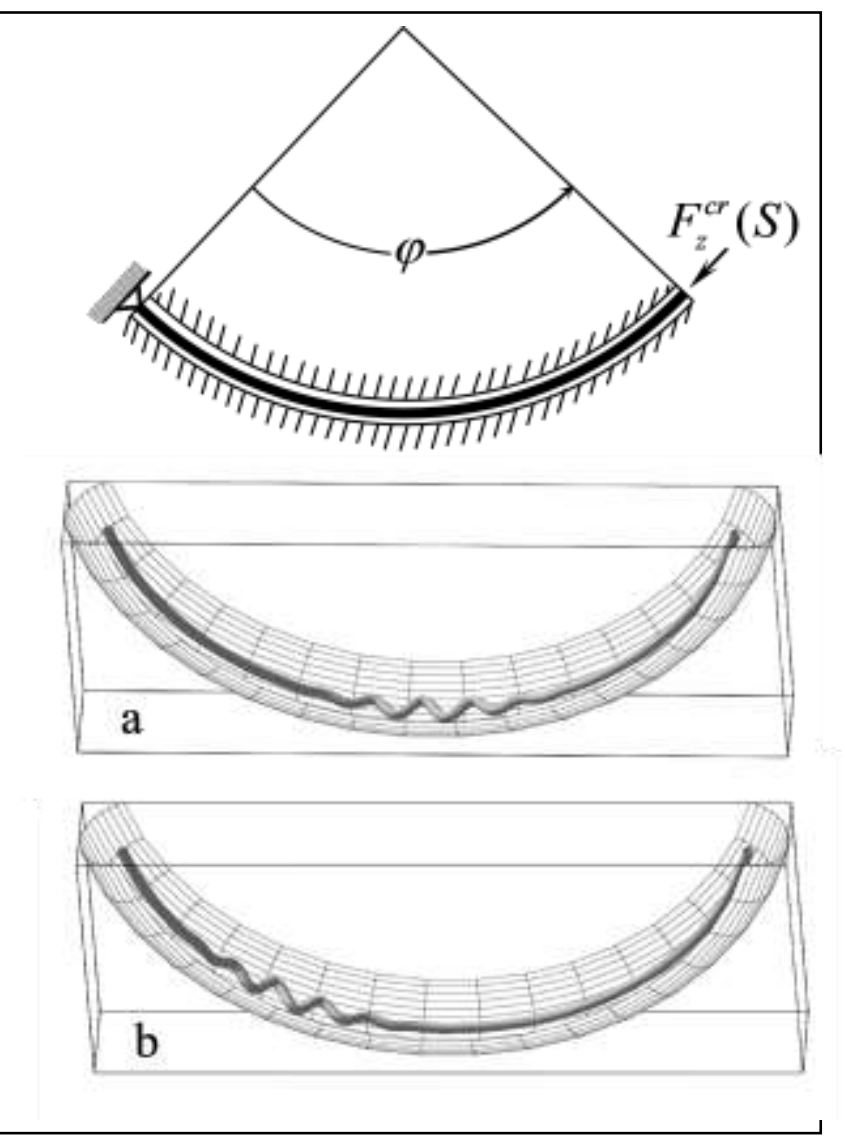

Figure 5. Drill string buckling in the symetric circular channel

Positions "a" and "b" in these illustrations correspond to the cases of frictionless and frictional motions. One can see that the influence of the friction forces on buckling modes is appreciable. They cause not only the diversification of the buckling zone positioning but lead to these zones widening as well. Besides, in the every zone limits, the pitches of conventional harmonics become also variable.

It is intriguing to compare the obtained results with the analytic solution deduced for the limiting case when the curvature radius $\rho$ tends to infinity in the absence of friction effects. Then, it is valid to assume that the rectilinear borehole is horizontal and the infinitely long DS is prestressed by the axial force $F_{z}(s)$ remaining unchanged throughout its length. In this event, the critical values of force $F_{z}(s)$ and pitch $\lambda$ are determined by equalities $[5,8]$

$$
F_{z}^{c r}=2 \sqrt{E I f^{g r} / a}, \quad \lambda^{c r}=\pi \sqrt[4]{E I a / f^{g r}} .
$$

It can be seen that the calculated results are closely related for large values of clearance $a$ and the difference between them grows with a reduction in $a$.

Summarizing obtained results, one can recognize that the found regularities of the realization of critical states and critical modes evolving are associated, in a large extent, with 
the circular geometry of a bore-hole and invariability of its curvature radius $\rho$. One might expect that the considered phenomena will be far more intricate for the wells with a variable curvature both in the cases of frictionless contacts and when the frictional interactions occur.

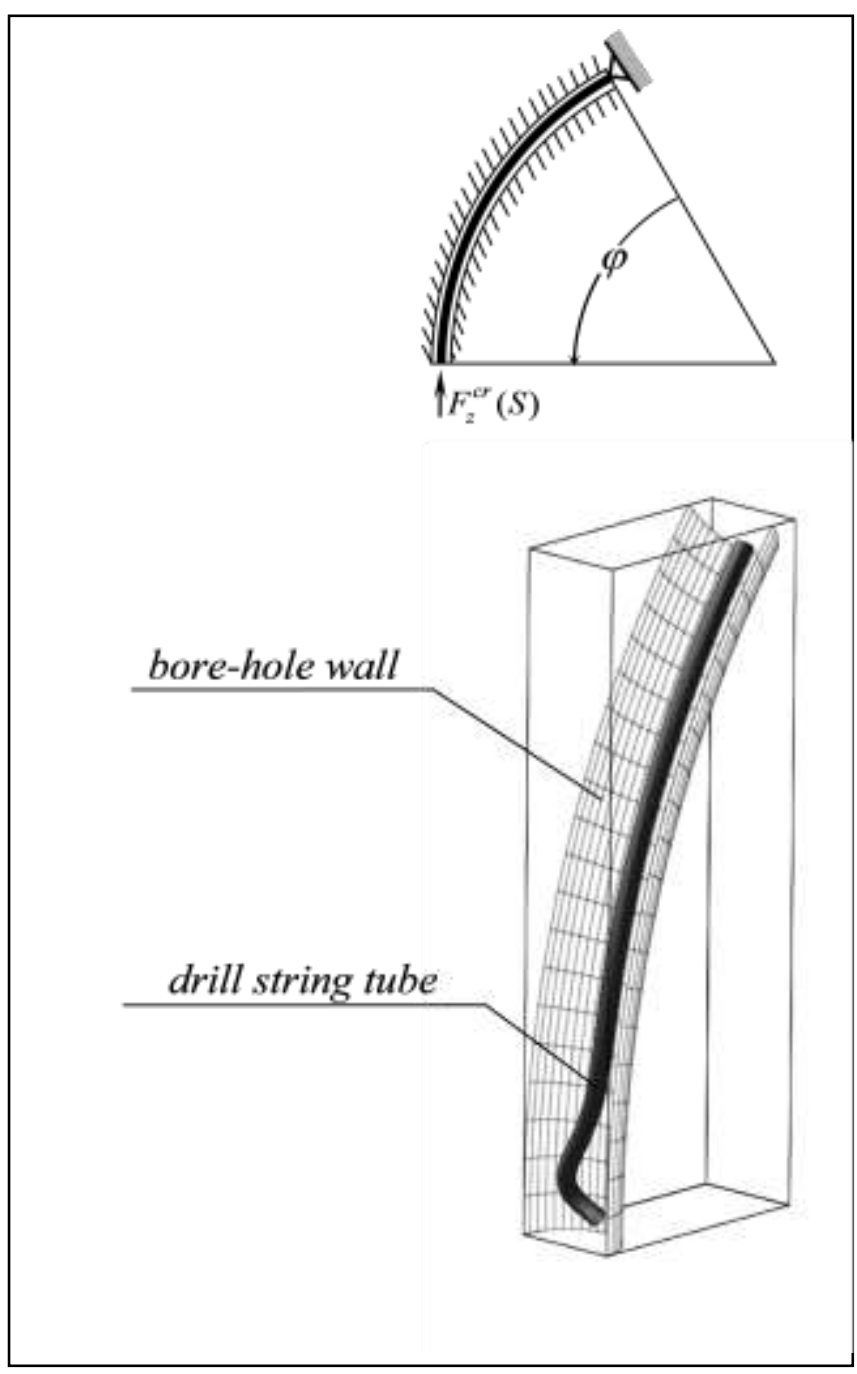

Figure 6. Drill string buckling in the symetric circular channel

Noteworthy also is the remark in relation to the influence of the bore-hole geometry imperfections on the buckling process. The geometry imperfections entail enlargement of distributed friction forces and axial force $F_{z}(s)$, on the other hand, the geometry distortions result in a change of the curvature radius $\rho$. Both these factors imply the essential effect on the buckling process and should be specially studied.

\section{References}

[1] B.S. Aadnoy, K. Andersen, "Design of oil wells using analytical friction models," Journal of Petroleum Science and Engineering, vol. 32, pp. 53$71,2001$.

[2] J. Choe, J.J. Shubert, and H.C. Juvkam-Wold, "Well control analysis on extended-rich and multilateral trajectories," SPE Drilling \& Completion, June, pp. 101-108, 2005.
[3] V.I. Gulyayev, E.N. Andrusenko, N.V. Shlyun, "Theoretical modelling of post - buckling contact interaction of a drill string with inclined borehole surface," Structural Engeneering and Mechanics, vol. 49, № 4, pp. 427-448, 2014

[4] V.I. Gulyayev, E.N. Andrusenko, "Theoretical simulation of geometrical imperfections influence on drilling operations at drivage of curvilinear bore-holes," Journal of Petroleum Science and Engineering, vol. 112, pp. 170-177, 2013.

[5] V.I. Gulyayev, V.V. Gaidaichuk, E.N. Andrusenko, N.V. Shlyun, "Critical buckling of drill strings in curvilinear channels of directed bore-holes," Journal of Petroleum Science and Engineering, vol. 129, № 1, pp. 168-177, 2015.

[6] V.I. Gulyayev, V.V. Gaidaichuk, E.N. Andrusenko, N.V. Shlun, "Modeling the energy-saving regimes of curvilinear bore-hole drivage," Journal of Offshore Mechanics and Arctic Engineering, vol. 137, № 1, pp. 011402-1-011402-8, 2015.

[7] V.I. Gulyayev, S.N. Khudoliy , E.N. Andrusenko, "Sensitivity of resistance forces to localized geometrical imperfections in movement of drill strings in inclined bore-holes," Interaction and Multiscale Mechanics, vol. 4, № 1, pp. 1-16, 2011.

[8] V.I. Gulyayev, N.V. Shlyun, "Influence of friction on buckling of a drill string in the circular channel of a bore hole," Petroleum Science, vol. 13, pp. 698-711, 2016.

[9] W.C. Lyons, G.J. Plisga, and M.D. Lorenz, "Standard Handbook of Petroleum and Natural Gas Engineering," Third Edition. Elsevier. Amsterdam, Boston, Heidelberg, London, New York, Oxford, Paris, San Diego, San Francisco, Singapore, Sydney, Tokyo, 2016.

About Corresponding author:

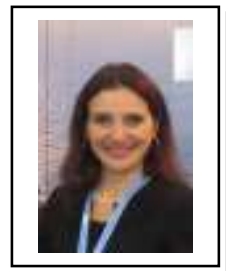

[Olena Andrusenko is at the moment a senior lecturer at the Department of Mathematics of the National Transport University. She was born in Poltava region (Ukraine). She graduated from the Department of Differential Geometry of the Kharkov National University in 2003 and she obtained her $\mathrm{PhD}$ degree in 2014.

Directions of her scientific activity are associated with the problems of mathematic simulation of drill-string deforming in hyper deep curvilinear boreholes and analysis of their emergency states.] 